\title{
La irrupción de una nueva ola feminista: ¿La cuarta ola? ${ }^{1}$ The Irruption of a New Feminist Wave: The Fourth Wave?
}

\section{Doris Lamus Canavate}

Universidad Industrial de Santander, Bucaramanga, Colombia. dlamus25@gmail.com | https://orcid.org/0000-0002-9149-0177

Recibido: 15 de junio de 2020. Aprobado: 7 de septiembre de 2020. DOI: $10.25100 /$ lamanzanadeladiscordia.v15i2.9808

\section{Artículo de investigación}

\section{¿Cómo citar este artículo? / How to quote this article?}

Lamus Canavate, Doris. (2020). La irrupción de una nueva ola feminista: ¿La cuarta ola?

La Manzana de la Discordia, 15(2),1-29. doi: 10.25100/lamanzanadeladiscordia.v15i2.9808

\footnotetext{
${ }^{1}$ Artículo producto de investigaciones desarrolladas por la autora a partir de un proceso sistemático de información.
} 


\title{
Resumen
}

Este artículo explora la hipótesis del surgimiento de una nueva ola de feminismos de naturaleza global, basado en el monitoreo de varias coyunturas que han tenido lugar desde 2014. La primera sección describe las características de las crecientes movilizaciones que comenzaron en América Latina y luego se extendieron a casi todos los países del mundo, destacando sus particularidades. La segunda discute y analiza la noción de la ola y su uso en las genealogías feministas más conocidas, para indagar si se trata o no de una de una cuarta ola. Finalmente, se revisa la contradicción entre producción y reproducción social, no solo como una amenaza para el progreso de las sociedades modernas en términos de derechos, sino también como una oportunidad para que los movimientos sociales introduzcan transformaciones basadas en "la revolución de los cuidados". Palabras clave: Cuarta Ola, Movimiento feminista, Producción vs. Reproducción social, Crisis de los cuidados.

\begin{abstract}
This article explores the hypothesis of the emergence of a new wave of feminisms of a global nature, based on the monitoring of various conjunctures that have taken place since 2014. The first section describes the characteristics of the growing mobilizations that began in Latin America and then spread to almost every country in the world, highlighting their particularities. The second discusses and analyzes the notion of the wave and its use in the best-known feminist genealogies to then specify whether it is indeed a fourth wave. Finally, the contradiction between production and social reproduction is reviewed, not only as a threat to the progress of modern societies in terms of rights, but also as an opportunity for social movements to introduce transformations based on "the revolution of care".
\end{abstract}

Keywords: Fourth Wave, Feminist Movement, Production vs. Social Reproduction, Crisis of Care. 


\section{Introducción}

En 2010, el Instituto de Antropología e Historia (ICANH) publicó el libro titulado De la subversión a la inclusión: Movimientos de mujeres de la segunda ola en Colombia, 1975 2005, resultado de una investigación desarrollada como trabajo de tesis entre 2003 y 2007 , en el programa de Doctorado en Estudios Culturales Latinoamericanos de la Universidad Andina Simón Bolívar, Quito, Ecuador. Transcurrida casi una década desde su publicación,

y sin haber seguido sistemáticamente los procesos organizativos de mujeres y feministas de los que allí dimos cuenta, me he detenido, coyunturalmente, en los acontecimientos que se producen en ámbitos académicos y en el activismo feminista, muy visibles en estos tiempos en medios y redes sociales.

Si bien he procurado consignar algunas de ellas en artículos publicados en La Silla Vacía, que permiten fotografiar la coyuntura, esta vez creo necesario retomar algunas ideas que den cuerpo a lo que aquí propongo explorar: la emergencia de una cuarta ola del movimiento feminista y de mujeres, con un conjunto de rasgos que han permitido a diversas autoras de distintos lugares del mundo, reiterar la trascendencia del momento feminista que está viviendo el planeta desde 2015.

Adicionalmente, como quedó planteado en aquel libro, es prudente anotar aquí que, cuando se indaga sobre estos y otros procesos, siempre quedan tareas pendientes, cabos sueltos, procesos inconclusos, preguntas más que respuestas sobre las cuales volver en cualquier momento. Retomo entonces algunos de aquellos cabos sueltos para examinar si efectivamente estamos ante una nueva, renovada, oleada feminista en el mundo.

Por su perspectiva latinoamericana, captó mi atención esta idea en la intervención de Francesca Gargallo en encuentro en la Universidad Simón Bolívar, en Barranquilla, Colombia (2019). Esta y otras fuentes en textos, blogs, revistas, artículos, videos, latinos e iberoamericanos, básicamente, quedan citados en este artículo. La producción es significativa y la tesis central que se extrae del conjunto de textos es que se observa la emergencia sostenida y global de movilizaciones sobre las que hay que elaborar contextos y teorías, así como hacer trabajo en terreno con protagonistas, porque todo parece indicar que estamos frente a una nueva ola, tal vez la cuarta, según cómo contemos las anteriores, y si entendemos estas como hitos históricos, no coetáneos, no simultáneos, no subsiguientes, ni exclusivos. Esta es nuestra hipótesis de lectura. 


\section{Agitación feminista en el último lustro}

Nuestro punto de partida es pues la insistencia de algunas autoras en interpretar el activismo feminista de los últimos cinco años, como "nueva ola", "cuarta oleada" o "la marea verde", al referirse a las enormes movilizaciones logradas en los países del sur del continente americano; a las igualmente multitudinarias, en el caso de Chile, con las protestas de las jóvenes universitarias contra la tolerancia institucional al abuso y al acoso sexual (Möller, 2018) o las de Argentina (Lospennato, 2018), a propósito de los debates por la legalización del aborto y que traen una continuidad desde 2015 con la campaña ¡Ni una menos! (Alcaraz, Gerez y Gordillo, 2019), o los paros (huelgas) nacionales e internacionales tanto en el continente americano como en el europeo (Gago, 2016), en el marco de la conmemoración del 8 de marzo (Lamus, 2018). Destacan en estas movilizaciones, la creciente presencia de jóvenes, mujeres en su mayoría, estudiantes de secundaria y universidades, su audacia desafiante y capacidad de convocatoria, entre otras características". "En la prensa la protesta ha cobrado visibilidad por la forma de manifestarse con los pechos descubiertos y los rostros enmascarados, pero el carácter autónomo, colectivo, sin huella de autoría personal de sus textos, es igualmente revolucionario" (Gargallo, 2019, p. 30), sostiene Francesca Gargallo.

Las jóvenes feministas se han juntado desde 2015 con sindicalistas y profesoras universitarias en el potente movimiento ¡Ni una Menos! Entre los años 2015 - 2019 las argentinas se han manifestado en masa contra los feminicidios, la trata de mujeres y la violencia contra niñas, mujeres y transgéneros, contra el Poder Judicial patriarcal que encubre violadores, redes de tratantes y feminicidas, y en favor del derecho al aborto. La enorme concentración de mujeres y hombres contra el feminicidio en Buenos Aires dio una sacudida a los desinflados movimientos feministas de España, Italia y Francia que, a su vez, provocó que el 8 de marzo de 2018 salieran a manifestarse feministas en todos los países de América, Europa occidental, norte de África, India, Tailandia y Australia. La misma huelga laboral, educativa, de consumo y de cuidados del 8 de marzo, se origina de las

\footnotetext{
${ }^{2}$ Mientras preparábamos este escrito, ha tenido lugar una masiva manifestación en Ciudad de México, ver artículo de Sonia Corona (2019) en el pais.com del 25 de agosto de 2019 y "Violaciones en CDMX" en bbc.com 13 de agosto de 2019 (BBC New, 2019). Durante el 2020 y en medio de la pandemia, las jóvenes mexicanas se han manifestado radicalmente contra los asesinatos de mujeres que crecen día a día (Villegas, 2020)
} 
concentraciones por la demanda de ponerle fin a la violencia feminicida, pero produce movimientos hacia el desplazamiento de los deseos de las mujeres hacia la libertad de movimiento, de palabra y expresión. (Gargallo, 2019, p. 28)

Gargallo ubica este proceso en el último quinquenio (2014-2019) y su notoriedad e inicio esta vez en Latinoamérica — no en USA o Europa, como las precedentes-, destacando el contexto local y las peculiaridades de las demandas feministas y de mujeres organizadas por la defensa de sus derechos. Y, pese a que en un principio discute el origen de la metáfora oceánica, las olas o mareas, cuya procedencia atribuye a los medios de comunicación, finalmente acepta y asume ese criterio en el desarrollo de su escrito: "Las metáforas oceánicas no están equivocadas cuando de feminismo se trata” (2019, p. 4).

Por su parte, y desde Colombia, Lorena María Aristizábal Farah (2019) sostiene en voz alta: "Ya somos marea" y desarrolla su aporte hacia una caracterización del movimiento feminista hoy. En su trabajo, incorpora una bien documentada información de prácticamente todo el planeta y que describe la agitación ocurrida en el mismo período del que se ocupara Francesca Gargallo. Parte de esta documentación está sustentada en el Informe 2017-2018 de Amnistía Internacional sobre la situación de los derechos humanos en el mundo. Sostiene que el feminismo está viviendo una nueva etapa, de alta visibilidad y aceptación (2018, p.266) (contrario a lo que ocurrió por décadas, hoy muchas mujeres se dicen feministas), la cual demanda una caracterización actualizada y es su propósito contribuir en ese sentido.

Parte la autora de reconocer la heterogeneidad cultural y política de los feminismos latinoamericanos, así como la de sus luchas y sus contextos, para reunir en cuatro áreas problemáticas y reivindicativas, un conjunto de asuntos que están y han estado desde el siglo pasado en la agenda feminista regional y global y que - dice-, hacen posible las articulaciones y las conversaciones que sostenemos, estratégicamente, académicas y activistas. Ellas nos permiten ahondar en las reflexiones que están emergiendo sobre cómo transformar algunas situaciones que enfrentan las mujeres y que recogen sintomáticamente las reivindicaciones y propuestas del momento feminista actual, sostiene Aristizábal (2019, p. 3). Tales asuntos son: el primero, las acciones de denuncia y movilización en torno al acoso sexual y los feminicidios; el segundo, la lucha por los derechos sexuales y 
reproductivos; el tercero, las reflexiones de las corrientes antirracistas y decoloniales y su crítica al feminismo hegemónico institucional y, el cuarto, las conversaciones en torno al reconocimiento y redistribución de los cuidados.

¿Qué se puede destacar hasta este punto de la exposición? Primero, es fundamental subrayar la existencia de una agitación feminista planetaria, sostenida y sin precedentes, asunto que se logra en contextos hoy conectados por las nuevas tecnologías de la información y las comunicaciones, no solo como medio, sino en tanto lugar de agitación conquistado por un nuevo ciberfeminismo; así mismo, es importante señalar que mientras las antiguas oleadas nos llegaban del norte, de Europa y Norteamérica, esta vez la mareada se origina en el sur de nuestro continente. Al respecto, Danila Suárez Tomé (2018) comenta:

Esta nueva ola que, a diferencia de las anteriores, se origina en los países latinoamericanos y se expande hacia el norte global, favorece también a la producción y difusión de las teorías feministas del sur, abriendo la posibilidad de una producción de conocimiento feminista más plural y descentrada. (2018, párr. 2)

De igual manera, otra característica sobresaliente que subrayo en esta oleada es que se evidencia una repolitización del discurso y las prácticas feministas, una oleada que se reconoce feminista sin disimulo, ni timideces y hasta sin miedo, aunque en buena medida toda esta agitación sea una respuesta a un cúmulo de tragedias históricas, como feminicidios, desapariciones, violencia de toda clase y violaciones de derechos humanos individuales y colectivos. Como lo recalca Lorena Aristizábal (2019),

Durante 2017 y 2018, la palabra feminismo se tomó las redes y medios de comunicación. En 2017, en Estados Unidos, la Editorial Merriam-Webster eligió el término como «Palabra del Año». La Marcha de las Mujeres que se tomó las calles de Washington y otras ciudades del país en reacción a la posesión del presidente Donald Trump, quién durante su campaña había expresado abiertamente opiniones machistas y propuestas adversas a los derechos de las mujeres, impulsó la búsqueda del concepto en páginas de internet y catapultó su popularidad. Los medios registraron que con respecto a 2016, la popularidad del término se incrementó en un $70 \%$. (EFE-Washington, 2017). (p.4) 
Es, además, de un fenómeno global, de masas (Toca, 2019), en el que mujeres en los cinco continentes, saudíes, turcas, palestinas, africanas, asiáticas, levantan la voz, llenan las calles y cruzan territorios con cadenas humanas ${ }^{3}$, demandando igualdad de derechos y contra la discriminación religiosa, como en India. También es un fenómeno intergeneracional, pues si bien los primeros lugares de la movilización en calles y plazas los ocupan desafiantes y furiosas mujeres jóvenes y adolescentes, y son las abanderadas del ciberfeminismo, la experiencia, reflexiones y la producción intelectual de las veteranas, complementa y conecta el trabajo del feminismo hoy. Se está consolidando un proceso de rearticulación del sujeto político del feminista (Cobo, 2019) tan duramente cuestionado en décadas pasadas y aún amenazado en tiempos presentes.

\section{La noción de olas en la construcción de genealogías feministas}

Las mujeres que hemos vivido en estos dos últimos siglos somos muy afortunadas por haber presenciado enormes transformaciones, revoluciones y desarrollos en distintos campos de la ciencia, la cultura y la tecnología, principalmente por haber participado, como protagonistas, desde los tempranos años del siglo XX en aquellas transformaciones que se relacionan con las condiciones de vida, sociales y jurídicas de las mujeres. Este protagonismo femenino en todos los campos y a nivel global traspasa el siglo XX. Los desafíos son monumentales, pero todo parece indicar que el siglo XXI es el siglo de las mujeres.

se van a lograr grandes cosas para las mujeres -y por consiguiente para el planeta-, en materia de legislación y conquista de derechos que son importantes, sí, en tanto propician una ruptura con el statu quo, con el peso de unas tradiciones, costumbres y prácticas que por siglos han pretendido mantener a las mujeres, las niñas y los niños, sometidos al poder de otro u otros, porque sí, porque Dios así lo quiso, o porque así ha sido de generación en generación. Pero, fundamentalmente, hay que insistir en transformaciones en términos de concepciones, de mentalidades y de prácticas congruentes con el reconocimiento de la condición de sujeto político, libre, autónomo, de cada una de las mujeres. (Lamus, 2018, parr 12)

\footnotetext{
${ }^{3}$ Así se registra en diferentes medios de comunicación como RT de enero 21 de 2019 y RED+ de enero 4 de 2019. 
Algunas filósofas, historiadoras y, en general, teóricas del feminismo, han llamado olas a estos procesos en los que se van construyendo genealogías a partir de las demandas y conquistas de las mujeres, en particular de las feministas organizadas en movimientos, sin que haya o tenga que haber acuerdo sobre el uso o no de esta taxonomía, entre otras cosas porque las historias que se cuentan allí corresponden a distintos tiempos, lugares, culturas y contextos. Es decir, se ha construido con esta narrativa una "metáfora oceánica", como dice Francesca Gargallo, por cuanto -y es mi comprensión del asunto-, tal como pasa con las olas del mar en su recorrido por el planeta, llegan en distintos tiempos y con mezclados contenidos, a cada orilla de la geografía planetaria, pero, a su vez, en cada lugar se encuentran con ríos, grandes o pequeños que le hacen aportes y hasta le cambian el color o la fuerza (la "marea verde").

Me gusta la noción de olas. La utilicé en el análisis de los movimientos de mujeres y feministas de Colombia (Lamus, 2010) con el sentido que le dio Agnes Heller (1988) para referirse a los movimientos culturales modernos en Europa y que incluye la idea de cresta que, figurativamente, marca el punto más elevado de la oleada en el tiempo, así como el descenso y la vuelta a emerger de la siguiente que, en ese proceso envuelve otras aguas, deja atrás aquellas de la cresta previa, aunque siempre trae algo de ella a la nueva, y así sucesivamente. Algunas activistas han incluido la figura del tsunami. En todo caso, aprecio el sentido dialéctico que conlleva esta metáfora. Dicho brevemente, puede resultar simple, pero de eso trata también la construcción de categorías analíticas: simple pero potente. No en vano ha sido el criterio básico en la construcción de algunas de las más conocidas genealogías del feminismo en Occidente.

¿Cómo, entonces, definimos el orden y el número de las “olas”? ¿Estamos en presencia de una nueva ola feminista, la cuarta, tal vez?

Entre el trabajo de teóricas y activistas se ha construido una genealogía muy importante que destaca por lo menos tres grandes olas, más o menos comunes a todos los países de Occidente y occidentalizados, aunque no sean precisamente coetáneas. Sin embargo, tiempo y contextos, así como especificidades culturales, sociales, económicas y políticas, interactúan con las aspiraciones de organizaciones y movimientos de mujeres y feministas, de modo tal que en las agendas van emergiendo, además de aquellas luchas generales y compartidas en alguna medida, otras que solo dan cuenta de historias, 
problemas o cuestiones locales o nacionales (por ejemplo, en el caso colombiano, hemos arrastrado década tras década el lastre de la violencia política, el conflicto armado, los escasos y pequeños momentos de paz, junto con reivindicaciones de la agenda feminista global).

Luego, es muy posible que en un relato de corte histórico general enunciemos y reconozcamos la existencia de tres olas, cuyo punto de partida está condicionado por el lugar de enunciación. Para las historiadoras, filósofas y feministas europeas, la primera etapa la ubican en el siglo XVIII, y se reconoce como el Feminismo Ilustrado (Amorós y De Miguel, 2005) en el que ya se cuestionaba el problema de la desigualdad entre los sexos, la racionalidad y la condición de subordinación de las mujeres. Como sostiene Celia Amorós y Rosa Cobo (2005) "el feminismo moderno parte de esta época y es el hijo no reconocido de la Ilustración". Por consiguiente, para las feministas españolas, la segunda ola es el momento sufragista, y la tercera el de los feminismos contemporáneos que parten de los años 70 del siglo XX. No obstante, otras autoras españolas, con otros marcadores y criterios, cuentan esos inicios del feminismo ilustrado junto con los de la búsqueda de reconocimiento jurídico y de ciudadanía (sufragismo) en una primera etapa, en tanto que la segunda correspondería a las décadas 1960/1970 y a lo que se agrupa bajo la denominación general de feminismo de la igualdad y la diferencia. La tercera, va de la década de 1990 en adelante y contiene el feminismo postmoderno, postcolonial, feminismo queer, ciberfeminismo y activismo radical (transfeminismo) (Rodríguez, 2015). Es decir, con unos y otros criterios, se reconocen tres olas.

En otros contextos, como Norteamérica y Latinoamérica, la primera ola, masiva, universal, sostenida en el tiempo (desde 1893, Nueva Zelanda) ${ }^{4}$ y conquistada, es el sufragismo; la segunda, el feminismo radical de la década de 1970, con origen en los EE. UU y, la tercera, el feminismo posmoderno y postcolonial de los años 80/90 del siglo XX. Sin embargo, como señalaba antes, depende del lugar de enunciación y del proceso que nos ocupemos. Para el caso de la historia del feminismo desde la perspectiva europea, aunque no sea "nuestra" historia, es relevante el punto de partida del feminismo moderno/ilustrado en tanto hito histórico, como proceso que da cuenta del largo recorrido de la lucha por la

\footnotetext{
${ }^{4}$ El medio de noticias La Red 21 (2009) de Uruguay en su sitio web reseña el listado de países con sufragio femenino con la fecha respectiva de aprobación.

La Manzana de la Discordia • Vol. 15. No. 2 • junio-diciembre $2020 \bullet$ pp. 1-26 • E-ISSN 2500-6738
} 
emancipación y los derechos de las mujeres, aunque no fuese un fenómeno masivo, ni global, sí como punto de partida de dos siglos largos de lucha, que dan origen al feminismo como movimiento social y político y como paradigma de conocimiento desde una visión no falogocéntrica.

Así mismo, para el norte de América y Europa (y tal vez más recientemente en Latinoamérica), buena parte de las visiones críticas postmodernas y postcoloniales feministas corresponden a la tercera ola, si contamos el sufragismo como la primera. Esta tercera ola del feminismo se desarrolla en los años 80 y 90 del siglo XX, y tiene su inicio en los planteamientos del feminismo cultural norteamericano. Son protagonistas las mujeres negras, chicanas, lesbianas y los transexuales. Y se sustentan en las corrientes teóricas radicales, marxistas y socialistas, junto con aportes del posestructuralismo: feminismo posmoderno y poscolonial (Dietz, 2005). Pero, insisto, dependiendo del criterio que se utilice (epistemológico/teórico, político, cronológico, entre otros), podríamos argumentar que los inicios de posturas como, por ejemplo, el feminismo negro (black feminism), emergen en el mismo contexto del feminismo radical de la década de 1970, de modo tal que esta oleada va creciendo y conduciendo, en las décadas siguientes, a la emergencia de nuevas generaciones que se posicionan frente a las diferencias étnicas y raciales, también frente a la heteronormatividad obligatoria.

Es así como en el desarrollo de los debates feministas ha habido siempre una cierta crítica interna, en el sentido de reflexiones y debates producidos en el interior de los propios grupos, sus postulados teóricos y epistemológicos y sus prácticas políticas. Este elemento de diferenciación es mucho más evidente en la transición hacia el pensamiento postmoderno y postcolonial, y las críticas se han tornado más radicales y "separatistas". Se ha escrito mucho al respecto, pero es un buen ejemplo el trabajo de Carolina Meloni (2012) titulado Las fronteras del feminismo. Teorías nómadas, mestizas y postmodernas. Tales desarrollos teóricos y epistemológicos van a tener un papel clave en la definición del punto de quiebre de cada ola. Así pues, en la medida en que la producción teórica (en general y de las feministas en particular) se hace más densa y, tal vez, más crítica, y las experiencias de mujeres de distintos lugares y culturas se confrontan con el sistema dominante y con "las otras" (blancas, burguesas, occidentales, heteronormativas, hegemónicas y un largo etc.) se 
hace también más difícil y casi inútil seguir el curso de las corrientes que conformarían las olas $^{5}$.

Como he venido sosteniendo, han existido ciertos hitos históricos que se hacen visibles en momentos de ruptura, de quiebre en las agendas de reivindicaciones feministas, en las consignas y las consiguientes críticas (discursos y prácticas) entre las precedentes y las siguientes (suponiendo una suerte de sucesión que no es tan precisa) que bien pueden ser el criterio de distinción. Estos hitos históricos o marcadores de una periodización son las así llamadas olas, y cobran sentido en eventos de larga o mediana duración, pero pueden resultar insuficientes o impertinentes en el análisis de ciertas coyunturas o eventos de reciente ocurrencia.

El caso de la ola sufragista es bien ilustrativo. Sin embargo, ninguno de los hechos que han dado nombre al hito histórico ha sido único o exclusivo; junto con los intereses de las mujeres por el derecho al sufragio, estaban sus intereses de clase (también su origen racial) y su filiación política: comunistas, socialistas, liberales, anarquistas y, sin duda, los contextos culturales y políticos de los países en que ellas planteaban sus demandas. Del mismo modo, ninguna de las olas que reconocemos hoy se ha extinguido, más bien ha tomado nuevos e importantes causes -recuerda mi descripción de la noción de ola-.

Así, volviendo a la mareada sufragista, esta tuvo su inicio, su desarrollo, su cresta y con ella casi todas las mujeres del mundo adquirieron su mayoría de edad; no obstante, en el camino del logro de derechos políticos y de conquistas de orden jurídico y constitucional, las mujeres siguen luchando por el reconocimiento de su derecho a ocupar posiciones de representación/elección/designación política en condiciones de igualdad/paridad, tarea en las que una buena proporción del activismo feminista trabaja permanentemente. Esa sigue siendo una corriente definida que ha cruzado los mares del tiempo, pero que es hija de esa primera ola sufragista.

En este mismo sentido, la llamada segunda ola también es identificable en su inicio y en confrontación con el sufragismo, ahora visto como elitista y clasista. La segunda ola de la década de 1970 se reconoce por su postura radical y su crítica al sistema de

\footnotetext{
${ }^{5}$ Cuando emergen diferencias tan radicales y estas se multiplican, el uso de la noción de ola como marcador de un hito es, al parecer, insuficiente. Sirve, más bien, para hacer un cierto corte cronológico/local, por ejemplo, pero es mucho más relevante, en el sentido del análisis de los movimientos sociales y sus particularidades, destacar las diferencias, el debate de fondo en la construcción de esas diferencias.

La Manzana de la Discordia • Vol. 15. No. 2 • junio-diciembre $2020 \bullet$ pp. 1-26• E-ISSN 2500-6738
} 
dominación patriarcal, sustentado en relaciones de poder que se afianzan en la diferencia sexual. Pero, junto con estas corrientes emergen otras igualmente radicales, que sustentarán sus reivindicaciones en otras diferencias más allá de la sexual e inaugurarán las reivindicaciones por la diferencia frente la igualdad, bandera esta de las sufragistas.

Y lo que se va a observar entre los años 70 y 90 del siglo XX es la conformación de corrientes de pensamiento y acción política feminista que construyen su propia agenda y frentes de lucha, ahora con un fuerte contenido identitario ${ }^{6}$. Cada década plantea unos debates, unas posturas, unas críticas a sus predecesoras que, con ayuda de las teorías que se elaboran en otros campos no feministas, pero ante todo de su uso crítico, enfrentan las encrucijada y dilemas de estos "nuevos feminismos" que, a veces, parecieran perder de vista con facilidad el objeto de su propio proyecto. Si queremos dar a esta tercera ola, de origen europeo y norteamericano, una etiqueta que marque las rupturas, la que más se ajusta a su diversidad sería la de los feminismos "post", sin desconocer la pervivencia de otras corrientes como la liberal y sus reactualizaciones.

A riesgo de ser impertinente, sostengo aquí que, lo que hemos tenido desde los años 80/90 del siglo XX, al menos en Colombia, son oleadas de la primera (sufragismo) y la segunda (feminismo radical) que se dispersan por nuestra geografía, historia y cultura. En Colombia, si bien se producen unos drásticos cambios que podrían sugerir el inicio de una tercera ola, no lo es por lo menos en el sentido de los feminismos del Norte. En el contexto colombiano y latinoamericano se reconoce para el período en referencia, el avance de un marcado proceso de institucionalización de los movimientos de mujeres y feministas, de corte democrático liberal, lo que configura el predominio de enfoques de tipo tecnocrático, desde el Estado, de participación política y formas de incidencia en políticas públicas (Lamus, 2010); con la lente de las olas, estaríamos en una etapa renovada, fortalecida e institucionalizada de un feminismo de inspiración liberal ${ }^{7}$.

\footnotetext{
${ }^{6}$ Estas corrientes de pensamiento se generan a partir de la década de 1980 con los debates posmodernos, posestructurales y poscoloniales, que desde la crítica interna del feminismo se expresarían en las corrientes de "la diferencia social", en el caso de las feministas norteamericanas que buscan revalorar la experiencia de las mujeres, y en el de la "diferencia sexual" europea, la cual produjo un complejo grupo de posturas conocido como feminismo francés, el cual tiene en común con el feminismo deconstruccionista la idea de que la identidad sexual es un fenómeno mediado por el discurso. Pero las de "la diferencia" cuestionan también el término mujer por universal y “esencialista” (Lamus, 2010, p. 24-25).

${ }^{7}$ La revisión de los procesos a partir de la década de 1970 en Colombia nos permitió sostener el argumento de una transición del discurso radical, subversivo de aquellas décadas a formas reivindicativas y políticas de La Manzana de la Discordia • Vol. 15. No. 2 • junio-diciembre $2020 \bullet$ pp. 1-26 • E-ISSN 2500-6738
} 
Se ha documentado suficientemente el proceso de institucionalización que vivimos en Colombia y en el resto de los países del continente, más en unos que en otros, en el que se destaca el predominio de un cierto feminismo liberal (Mainstream o corriente mayoritaria de pensamiento; o "feminismo hegemónico", según quien interprete), con mujeres muy bien formadas en universidades y centros de investigación, justo con el intenso proceso de ONGización de los movimientos sociales feministas y de mujeres ${ }^{8}$. Otro elemento destacado de este período fue la incorporación de la categoría género ${ }^{9}$ en el discurso y las prácticas de las ONG, de la academia feminista y en muy diversos espacios como los medios de comunicación y el Estado y sus políticas para la equidad de género, ganadas por los movimientos.

Iniciado el siglo XXI se retoman en Latinoamérica, bien sustentados en proyectos académico-políticos en la región ${ }^{10}$, las reivindicaciones identitarias que ya habían hecho carrera en Norteamérica con las migraciones de intelectuales de los países que recién se liberaban del yugo colonial. Mirado con la lente de las olas, en mi particular lectura, sería el ingreso de discursos y prácticas feministas de inspiración "post", en confrontación, primordialmente, con el denominado "feminismo hegemónico". Así, junto con las posturas postmodernas y las postcoloniales se abrirán nuevos caminos a la reflexión ahora en clave decolonial. En Colombia, sin embargo, en el contexto de confrontación política y violencias cada vez más complejas, seguimos impulsando una agenda que pretende avanzar en medio de esa maraña, de modo que tales debates son, por lo menos, recientes, pero ya está instalado en el debate regional la crítica decolonial que encierra unas posturas antiracistas, anti-capitalistas y anti-patriarcales, principalmente.

estirpe liberal-institucional, lo que dio origen al título de la publicación: De la subversión a la inclusión, movimientos de mujeres de la segunda ola en Colombia, 1975-2005 (2010).

${ }^{8}$ En el trabajo al inicio citado y aquí he utilizado esta denominación por cuanto las estudiosas de los movimientos sociales han hecho la distinción entre el movimiento de mujeres, que no son necesariamente feministas y se supone más amplio, y el movimiento feminista propiamente tal que defiende una agenda más o menos compartida. Sin embargo, en su interior, la diversidad no es solo referida a todas las aquí mencionadas, sino que algunas dicen defender los derechos de las mujeres y no ser feministas, o que trabajan por "la transversalización del enfoque de género", pero no son feministas, o que son feministas con algún apellido, al menos en lo que hemos conocido a través del trabajo de investigación. Queda, sin embargo, por profundizar la tarea de discernir más detenidamente las diferencias.

${ }^{9}$ Los debates alrededor del uso de la categoría analítica género no terminan, pero el efecto de despolitización y repolitización del concepto y sus implicaciones para el proyecto feminista están también por decantar (Lamus, 2012).

${ }^{10}$ A manera de ejemplo, de gran influencia en la región, cito grupo de investigación "ModernidadColonialidad" (Escobar, 2003).

La Manzana de la Discordia • Vol. 15. No. 2 • junio-diciembre $2020 \bullet$ pp. 1-26 • E-ISSN 2500-6738 
Existen así mismo un conjunto de debates que, a mi juicio, bajo las premisas o denominaciones de postcoloniales y postmodernas quedan groseramente desdibujadas porque no se corresponden con contextos y procesos no solo distintos a los propios, sino sencillamente ajenos a ellos. Estoy pensando en los así denominados hoy feminismos comunitarios, pero siendo un poco más flexible, en las muy diversas formas organizativas que han surgido de las mujeres indígenas de México, Centroamérica y de los países del continente con amplia presencia indígena/originaria. Este sí, un hecho histórico, pues sus orígenes son otros, pero de muy reciente atención y visibilidad para analistas externos o por personas originarias ${ }^{11}$, que ya ha empezado a dar importantes frutos en términos académicos y políticos. Solo como ejemplo, cito aquí una didáctica construcción de una genealogía de los feminismos presente en el trabajo de la Escuela Política Feminista de Guatemala (Asociación Feminista La Cuerda, Asociación de Mujeres de Petén Ixquik, Alianza Política Sector de Mujeres, 2010), el cual incorpora buena parte de la producción de occidente desde las ilustradas, con un criterio de partida: por un lado, los feminismos "de la igualdad" y por el otro "los de la diferencia". Sin desconocer que este esquema contiene limitaciones, es una buena herramienta para el trabajo pedagógico y de formación política que pretenden. El esquema permite incorporar lo que aquí hemos llamado olas, e incluir los feminismos indígenas/comunitarios propios del centro y sur del continente americano, así como las teorías queer.

No obstante, mi llamado de atención es hacia la pertinencia o no de pretender meter todas las formas históricas adoptadas por los feminismos en el esquema de las $o_{\text {olas }}{ }^{12}$. Aquí sí hay que plantearse un reto y un gesto decolonial para hacer visibles estas luchas de las mujeres originarias, sin pretender hablar por ellas. Pero, este es un planteamiento aún en borrador, con más sospechas e ilusiones que con argumentos contundentes. Ha sido, de todos modos, siguiendo el vaivén de las olas que he llegado a plantearme este nicho de exploración. Una nueva tarea pendiente.

\footnotetext{
${ }^{11}$ Son reconocidas e influyentes las voces de Lorena Cabnal, de Guatemala, Adriana Guzmán y Julieta Preciado de Bolivia, entre otras, cuyos escritos, conferencias y videos han sido muy útiles en el trabajo pedagógico. Ver: Doris Lamus Canavate (2020). “Genealogía de los feminismos”, Escuela de Formación Mujer, Poder y Política, FMF-HBS. Bucaramanga.

${ }^{12}$ Las categorías que nos resultan útiles y dinámicas no tienen por qué pensarse como universales e infinitas. Son tan aplicables como puedan ser, sin pretender extraer de ellas más de lo que es posible. Este es el sentido del análisis de los asuntos humanos, sociales, culturales y políticos.

La Manzana de la Discordia • Vol. 15. No. 2 • junio-diciembre 2020 • pp. 1-26・E-ISSN 2500-6738
} 
En resumen, sea con el criterio de las filósofas españolas, o con el criterio nortelatinoamericano, son tres las olas identificadas de modo general en las genealogías del feminismo como postura teórico-política y como movimiento social. Luego del ejercicio realizado, y habiendo puntualizado a lo largo de este ensayo las "virtudes" y las limitaciones de la noción de ola, debo subrayar su pertinencia en estudios de larga y mediana duración. En nuestro caso, ha permitido avanzar en esta reflexión y da cuenta de tres siglos de feminismos en Occidente y regiones occidentalizadas. Esta permanencia en el tiempo es un criterio clave para identificar un movimiento social.

\section{De la producción a la reproducción social: crisis periódicas del capitalismo y} la crisis de los cuidados

...la globalización es especialmente catastrófica para las mujeres

y no porque la controlen agencias dominadas por hombres, que no son conscientes de las necesidades de las mujeres, sino por los objetivos que se ha marcado la globalización. Revolución en Punto Cero (Federici, 2013, p. 144)

Reconecto esta última sección con el último de los cuatro núcleos temáticos alrededor de los cuales Lorena María Aristizábal Farah (2019) elabora su tesis de que Ya somos marea; esto es, con el asunto del reconocimiento y la redistribución de los cuidados, y que expone como insoslayable en los cuatro puntos problemáticos en la agenda reivindicativa de los feminismos hoy, en tanto compete a la organización de la sociedad en su conjunto.

Con ello pretendo explorar esta hipótesis de lectura que ve, en la "crisis" que experimenta el sistema en las dimensiones de la reproducción social y las tareas de cuidado, una oportunidad para la reestructuración de tareas que han sido asignadas históricamente y naturalizadas como "propias de las mujeres", con las consecuencias de representar cargas laborales y sociales excesivas, y desigualdad estructural que se traduce en explotación, exclusión y subordinación, así como limitaciones/negaciones en las posibilidades y potencialidades de su propia existencia como ser humano y como sujeto político.

El contexto de fondo que diversos autores (Castells, 2017; Fraser, 2016; Piketty, 2014) vienen analizando es la existencia de esto que, desde Marx, la economía clásica ha identificado como crisis periódicas del capitalismo, la más reciente experimentada en 2007-2008, que se manifiesta en diversas esferas de la vida, no solo económica y 
financiera, con lo cual se hace mucho más tangible, también, su naturaleza estructural/sistémica.

Una perspectiva distinta en el análisis de estas crisis es la que están planteando autoras feministas, principalmente Fraser (2016), también Federici (2013), que subrayan la relación que intento seguir aquí: la agudización de la contradicción en el propio núcleo del sistema entre producción/reproducción social, lo cual ha sido discutido y criticado desde tiempo atrás por feminismos de orientación marxista o materialista, pero que, en el contexto de las más recientes movilizaciones feministas, por lo menos en Latinoamérica y en España, se plantea como oportunidad para avanzar en la agenda para fortalecer la lucha por la transformación en una perspectiva de la economía feminista (Rodríguez, 2018) y de posturas del ecofeminismo (Herrero, 2013).

Puntualicemos, en principio, la ubicación de esta reflexión en el contexto del régimen capitalista neoliberal en que vivimos y sus efectos más perversos en la reproducción social, entendida como núcleo de la actividad humana — remunerada y noen el cual se dan las formas más extremas de explotación de la población en su conjunto en la actualidad, ante todo de la población empobrecida y, en general, de la población femenina, si entendemos el sistema como sustentado en y producido por las desigualdades estructurales autogeneradas. Así mismo, en un horizonte más amplio de su globalización:

El objetivo primordial de la globalización es proporcionar al capital el control total, sobre el trabajo y los recursos naturales y para ello debe expropiar a los trabajadores de cualquier medio de subsistencia que les permita resistir un aumento de la explotación. Y dicha expropiación no es posible sin que se produzca un ataque sistemático sobre las condiciones materiales de la reproducción social y contra los principales sujetos de este trabajo, que en la mayor parte de los países son mujeres. (Federici, 2013, p. 144)

Así mismo, el argumento de Fraser et alt. (2019) sostiene que la supervivencia del sistema capitalista depende de la explotación del trabajo asalariado; pero, además, este se aprovecha de la naturaleza, de los bienes públicos y del trabajo no remunerado que reproduce a los seres humanos y sus comunidades. Las labores de reproducción social, particularmente las no remuneradas, son impactados por las crisis del sistema en general 
en la medida en que el capital desestabiliza permanentemente las condiciones mismas que él, la población y el planeta necesitamos para sobrevivir ${ }^{13}$.

Fraser (2016) llama a esta situación «crisis de los cuidados» y cree que es uno de los temas centrales del debate público y explica que esta expresión

hace referencia a las presiones que desde diversos puntos están actualmente exprimiendo un conjunto clave de capacidades sociales: las disponibles para tener y criar niños, cuidar de amigos y familiares, mantener hogares y comunidades más amplias, y sostener relaciones más en general. (Fraser, 2016, p.111) ${ }^{14}$

¿Quiénes experimentan estas presiones?

Históricamente, estos procesos de «reproducción social» han estado considerados trabajo de mujeres, aunque los hombres siempre han realizado también parte de ellos. Los cuidados, que comprenden tanto trabajo afectivo como material y a menudo se realizan sin remuneración, son indispensables para la sociedad. Sin ellos no podría haber cultura, ni economía, ni organización política. Ninguna sociedad que sistemáticamente debilite su reproducción social logra perdurar mucho. Hoy en día, sin embargo, una nueva forma de sociedad capitalista está haciendo exactamente eso. El resultado es una enorme crisis, no solo de los cuidados, sino también de la reproducción social en su sentido más amplio. (Fraser, 2016, pp.111, 112)

Esto es, crisis en el vital y complejo trabajo de hacer personas; vital, en tanto este trabajo crea y nutre la vida en su sentido biológico, y complejo, en tanto crea y nutre la fuerza de trabajo con las competencias, habilidades y destrezas necesarias para el sistema (Fraser et alt., 2019, p. 38).

Es posible jugar aquí con una hipótesis de lectura provisional de todo este proceso según la cual, en la misma medida en que el sistema intensifica la extracción de ganancias en muy diversos frentes y, simultáneamente, degrada, precariza y elimina condiciones de trabajo remunerado y no remunerado necesarias para la reproducción social, estos impactos son identificados y repensados por sectores críticos en la academia y el activismo, no solo

\footnotetext{
${ }^{13}$ El capital financiero migra frecuentemente buscando sitios donde pueda obtener mayores ganancias a menor costo. Con ello se profundiza la precariedad de los empleos, se desplaza a las poblaciones de sus territorios, explotan, degradan y agotan los "recursos naturales" y mercantilizan todos los aspectos de la vida. ${ }^{14}$ A continuación, cita una nutrida bibliografía que sustenta su afirmación. 
feministas, como una suerte de alerta máxima que lleva tanto a la acción política y la movilización masiva y planetaria ( 8 de marzo), como a rearticular la reflexión teórica y política de académicas y diseñadores/gestores de políticas sociales globales.

Es lo que se observa en la abundante literatura citada en los trabajos de las autoras aquí citadas. Así mismo, una rápida búsqueda evidencia como, además de la sensibilidad para captar la pertinencia y la importancia de este debate y de las acciones que se han puesto en práctica en América Latina, las preocupaciones por la "crisis de los cuidados" han sido analizadas también desde el punto de vista de las políticas sociales y las buenas prácticas identificadas por las agencias de cooperación internacional, el Sistema ONU y sus agencias en la región ${ }^{15}$.

En América Latina se trabaja en diversos frentes, particularmente con grupos y organizaciones de mujeres por la transformación de la esfera de la reproducción social. En este sentido, se reivindica el reconocimiento, la redistribución y la reducción de las labores de cuidado no remunerado. En Colombia, a partir de un trabajo de largo aliento de destacadas economistas y organizaciones de mujeres, se tiene la Ley 1413 de 2010 "Por medio de la cual se regula la inclusión de la economía del cuidado en el sistema de cuentas nacionales con el objeto de medir la contribución de la mujer al desarrollo económico y social del país y como herramienta fundamental para la asignación definición e implementación de políticas públicas". La ley ordena al Departamento Nacional de Estadística - DANE, la realización de una encuesta nacional cada tres años, sobre el uso del tiempo de las mujeres en el país, con el fin de identificar aquellas actividades no reconocidas ni remuneradas que, en una altísima proporción, realizan las mujeres dentro del hogar para atender y cuidar a su familia y su lugar de habitación (DANE, 2017).

En cuanto al activismo feminista, uno de los caminos aquí sugeridos por las autoras, retoma la experiencia acumulada por las feministas desde el siglo XIX, pero con la impronta de los nuevos tiempos en este siglo XXI: la reinvención y la ampliación de la huelga/el paro, como estrategia aglutinante y movilizadora, tal como se ha visto durante estos últimos cinco años en las calles y en las plazas de casi todos los países del planeta

\footnotetext{
${ }^{15}$ Ver, por ejemplo, Informe de investigación de OXFAM, de octubre 2013, titulado El cuidado en los hogares y las comunidades, Documento conceptual, el cual contiene una posición desde la organización y su directriz para sus cooperantes, así como una especie de estado del debate y evolución de lo que en este contexto llamamos trabajo de cuidados.

La Manzana de la Discordia • Vol. 15. No. 2 • junio-diciembre 2020 • pp. 1-26 • E-ISSN 2500-6738
} 
(Fraser et alt., 2019, pp. 20-21). Y aquí está la clave de esta "nueva forma de política" antes pensada solo desde lo sindical, desde la visión de la explotación del trabajo asalariado: la huelga feminista de hoy denuncia y amplía el ámbito de aquello que cuenta como trabajo. El trabajo de hoy, además del remunerado, es el trabajo no pagado. "Se hace así visible y manifiesto el rol indispensable que juega el trabajo no remunerado de las mujeres en la sociedad capitalista" (Fraser et alt. 2019, p. 21). Adicionalmente, esta estrategia de la huelga ha incorporado en la agenda de las movilizaciones, las demandas antiguas y recientes que han sido clave en la articulación: las acciones de denuncia en torno al acoso sexual y los feminicidios; la lucha por los derechos sexuales y reproductivos; los debates y agendas de las corrientes antirracistas y decoloniales y, a lo que aquí trabajamos con cierta relevancia: los asunto en torno al reconocimiento y redistribución de los cuidados, tal como se planteó al inicio (Aristizábal, 2019).

En resumen, el problema de la crisis de los cuidados y en general de la reproducción social de enormes contingentes de población bajo el régimen capitalista global neoliberal está ya en la agenda del movimiento feminista global, en el foco de las acciones de las agencias de cooperación, en las políticas estatales, cada cual con sus respectiva perspectivas e intereses. No obstante, el panorama es bastante más complejo y estas constataciones no pueden llevarnos a conclusiones apresuradas. Algunas miradas de reconocidas autoras nos aportan elementos para esta especie de diagnóstico.

Rita Segato (2018) ha planteado un asunto que complementa el mapa de los desafíos que confrontan los feminismos de hoy. A su juicio, es la democracia la que está siendo sitiada y adhiere a una cierta "teoría del complot", según la cual existen dos amenazas, de la misma potencia y peligrosidad, que están haciendo tambalear los avances de la sociedad en su conjunto: una, proveniente del crimen organizado o "la esfera paraestatal de control de la vida", del cual el objetivo central de su acción es el cuerpo de las mujeres: violaciones, feminicidios, asesinatos masivos. Y la segunda, proveniente de la emergencia de sectores católicos y evangélicos fundamentalistas de las iglesias que tienen el plan en ejecución de convertir a América Latina en un Medio Oriente por medio de la guerra religiosa, e implantar el miedo al fanatismo faccional religioso que se expresa también por el control de los cuerpos de las mujeres. 
Es un escenario sencillamente tenebroso. Y cada uno de los caminos seguidos en el análisis llega a la misma conclusión, la destrucción tiene como su objeto predilecto, a la vida misma y con ella el cuerpo de las mujeres. Adicionalmente, y desde una postura autocrítica, creo que no podemos perder de vista que mantenemos una multitud de diferencias, no solo de lecturas del contexto, sino de agendas y de apuestas políticas de tal complejidad que la tarea de encontrar los puntos de encuentro se vuelve central en la agenda y la estrategia de una eventual cuarta ola. Como afirmó Silvia Federici recientemente, en la clausura del Foro Social Mundial: "Es importante la unión de los movimientos porque la situación va a estar muy fea” (Crespo, 2020, parr. 1).

Como sostienen nuevas generaciones de mujeres de filiación anarquista, siendo hoy el feminismo mucho más "fragmentario y plural” (Libre pensamiento, 2017, p. 4), es de primer orden trazar una agenda y una estrategia que integre, que permita tener en ella las demandas por la igualdad, la lucha contra la violencia machista, la mirada de la economía feminista, las posturas antirracistas, el ciberfeminismo, las expresiones de la diversidad sexual. Claro está, sin olvidar o desestimar otras discusiones que están sobre la mesa, como la prostitución, el alquiler de vientres, y muchas otras formas de explotación agudizadas hoy por las guerras, las migraciones y el empobrecimiento de amplias regiones del planeta $^{16}$. En resumen, tenemos que aprender una nueva cultura política feminista que supere el separatismo porque el futuro nos exige acciones articuladas, inteligentes e imaginativas, porque "La revolución será feminista o no será".

\section{Conclusiones}

Este ejercicio de reflexión sobre algunas hipótesis de lectura elaboradas a partir de la observación de ciertas coyunturas de la movilización social feminista reciente, así como de la revisión de alguna literatura relacionada, es un tanto limitado como para pretender formular asuntos concluyentes. Antes, por el contrario, nos plantea la necesidad de la profundización, debate y desarrollo en terreno de muchas de las entradas posible al entramado implicado en los temas, problemas, categorías de análisis y perspectivas,

\footnotetext{
${ }^{16}$ Post scriptum. Si el panorama era tan amenazante en el momento de cierre de este artículo, habría que incluir en este rápido balance la situación planetaria que se enfrenta con el "Covid 19" y las consecuencias en las economías y en la reproducción social, tal como se aborda aquí.

La Manzana de la Discordia • Vol. 15. No. 2 • junio-diciembre 2020 • pp. 1-26・E-ISSN 2500-6738
} 
algunos de ellos, de orden estructural, particularmente en el asunto de la producción vs. reproducción social.

Sobre la llamativa hipótesis del tránsito, construcción o formación de una cuarta ola, he desarrollado aquí el ejercicio de poner a prueba mi propia visión y uso de la noción de olas. Y he de concluir que no solo resultó útil, sino que además me permitió identificar sus "virtudes" y sus límites. En este sentido, debo puntualizar algunos elementos.

En desarrollo de la narrativa de la "metáfora oceánica" para analizar los movimientos sociales feministas y de mujeres, uso la noción pensando en que, con ellos observamos unos procesos tal como pasa con las olas del mar en su recorrido por el planeta. Llegan en distintos tiempos y con mezclados contenidos, a cada orilla de la geografía planetaria; pero, a su vez, en cada lugar se encuentran con ríos, grandes o pequeños que le hacen aportes y hasta le cambian el color o la fuerza. Permite así, visualizar la cresta que, figurativamente, marca el punto más elevado de la oleada en el tiempo, así como el descenso y la vuelta a emerger de la siguiente que, en ese proceso envuelve otras aguas, deja atrás aquellas de la cresta previa, aunque siempre trae algo de ella a la nueva, y así sucesivamente, como vimos con el sufragismo.

También observamos con el dispositivo metafórico de las olas que, en el análisis de los movimientos sociales y en particular el de mujeres/feministas, tiempo y contextos, así como especificidades culturales, sociales, económicas y políticas, interactúan con las aspiraciones de organizaciones y movimientos, de modo tal que en las agendas van emergiendo, además de aquellas luchas generales y compartidas en alguna medida, otras que solo dan cuenta de historias, problemas o cuestiones locales o nacionales.

Fundamental para reconocer la existencia de hitos históricos como los que aluden las olas de los feminismos, es identificar el lugar de enunciación. Desde dónde hablo, con qué lente construyo una genealogía determinada y con qué apuesta política (puedo decir una apuesta "desde Europa" o "eurocéntrica", por ejemplo).

Están presentes, necesariamente, marcadores de tiempo y criterios (epistemológicos, políticos...) que van a depender, del lugar de enunciación y del proceso que nos ocupemos. Así mismo, la crítica interna y los desarrollos teóricos y epistemológicos y sus consecuencias políticas van a tener un papel clave en la definición del punto de quiebre de cada ola. 
Así entendida en mi ejercicio, es una noción útil, dinámica, flexible, pero tiene sus limitaciones que no dependen de su "eficacia", sino de la naturaleza y complejidad de los procesos que pretendemos observar y de la madurez de su desarrollo.

Entonces, sobre la primera pregunta de si ¿estamos transitando hacia una cuarta ola? me inclino a pensar que sí, que hay procesos en curso que tienen una serie de características con las cuales las teóricas ya están contando en tal escenario. Esto coadyuva a la mejor definición de su horizonte de sentido. Así como muestran las agendas la diversidad de acciones y tareas políticas en curso, está también la pregunta que a mi juicio debe orientar la reflexión y la acción y que, por supuesto, no es nueva, pero que, ante el escenario aquí dibujado, es perentorio: estrategias no solo de movilización juntas, sino de trabajo de articulación más a fondo, frente al riesgo inminente de mayor "fragmentación” y separatismo.

Una respuesta más contundente nos la dará el desarrollo de procesos en el tiempo y en los lugares donde las mujeres combaten de distinta manera no solo al patriarcado, sino a todos los otros males que nos amenazan, guerras, hambrunas, pandemias.

La segunda es, ¿qué posibilidades no solo de movilización, sino de transformación de condiciones presentes para las mujeres, ofrece trabajar como núcleo central de la agenda la problemática de la reproducción social y de los cuidados vs. la producción?

Es una buena apuesta y hay esfuerzos en esa dirección a nivel regional y en países que han vivido algo de aquel modelo de bienestar que se pensó el siglo pasado frente a la amenaza comunista, lo que seguramente hace una diferencia. Pero, siendo radicalmente consciente de las limitaciones de tiempo y espacio para responder a esta cuestión, creo necesario, al menos enunciar algunos elementos más bien de orden hipotético.

Si bien existen valiosos e importantes aportes que caminan en ese sentido, así como innumerables acciones que procuran desestructurar las relaciones que sustentan el orden patriarcal, no hay que perder de vista que este núcleo del cual nos hemos venido ocupando, es centro neurálgico del propio sistema, ese que prefiere destruir, agotar, degradar, contaminar, como si la vida y la naturaleza fuesen infinitas o, como es tal vez la idea, exprime hasta la última gota y va a otro lado por más, cual depredador incansable. En este sentido, esta dimensión de la agenda reivindicativa de los feminismos tiene que articular a esta una ruta ecofeminista de defensa del ambiente y el territorio ante al cambio climático, 
cuya confrontación no puede hacerse sosteniendo el modo de producción actual y su consecuente organización social de los cuidados (Herrero, 2013).

Tal vez estas estrategias puedan acercarse más a los feminismos comunitarios cuya defensa de la vida y el territorio, está necesariamente vinculada con el cuidado también del agua y de todo aquello que el capitalismo convirtió en "recursos naturales". No como modelo a imponer, sino como propuestas diversas orientadas a quebrar el espinazo a la bestia.

Si las condiciones de posibilidad se mantienen, y todo parece indicar que así será, la agitación de esta aún en construcción cuarta ola, combativa, militante, subversiva, va a continuar $^{17}$.

Es la tarea de académicas y activistas tomar conciencia de la naturaleza de las amenazas que conspiran contra las conquistas de dos siglos, pero también de las oportunidades que en estos momentos se pueden reorientar en nuestro favor. La pregunta es ¿hacia dónde?

\section{Referencias bibliográficas}

Alcaraz, María; Gerez, Majo y Gordillo, Florencia. (3 de junio de 2019). A cuatro años de Ni Una Menos: avances y retrocesos a nivel nacional, CABA, Córdoba y Rosario. Latfem. Disponible en: https://latfem.org/a-cuatro-anos-de-ni-una-menos-avances-yretrocesos-a-nivel-nacional-caba-cordoba-y-rosario/

Amnistía Internacional (2018) Informe 2017-2018 Amnistía Internacional. La situación de los derechos humanos en el mundo. Londres: Amnesty International Ltd.

Amorós, Celia y Álvarez, Ana de Miguel (Coord.). Teoría feminista: de la ilustración a la globalización. España: Editorial Minerva.

Amorós, Celia. y Cobo, Rosa. (2005). Feminismo e ilustración. En Celia Amorós y Ana de Miguel Álvarez (Coord.). Teoría feminista: de la ilustración a la globalización. (pp. 91144). España: Editorial Minerva.

\footnotetext{
${ }^{17}$ Post scriptum. La pandemia ha puesto a prueba también esta hipótesis. Por lo pronto, se agudizan las condiciones de existencia para millones de familias en el planeta, la solidaridad está a la vanguardia en las actividades de las organizaciones, pero, aún en medio de las restricciones de la pandemia, las movilizaciones se han producido en varios países, junto con otras causas. Seguiremos observando.

La Manzana de la Discordia • Vol. 15. No. 2 • junio-diciembre $2020 \bullet$ pp. 1-26・E-ISSN 2500-6738
} 
Aristizábal, Lorena. (2019). "Ya somos marea". Aportes para una caracterización del movimiento feminista hoy. Ideas Verdes, análisis político. (19) 1-25.

Asociación Feminista La Cuerda, Asociación de Mujeres de Petén Ixquik, Alianza Política Sector de Mujeres. (2010). Escuela Política Feminista: módulo 1: Genealogía feminista. Guatemala: La Otra Cooperativa/Editorial La Trilla.

Castells, Manuel. (2017). Ruptura: la crisis de la democracia liberal. Madrid: Alianza Editorial.

Cobo, Rosa [emakunde]. (18 de julio de 2019). Somos la cuarta ola. [Archivo de video] Disponible en: https://www.youtube.com/watch?v=yrug7GcNj2I

Corona, Sonia. (25 de agosto de 2019). El grito feminista retumba en México. El País. Disponible en https://elpais.com/sociedad/2019/08/24/actualidad/1566676851_265446.html

Crespo, Blanca. (19 de noviembre de 2020) Silvia Federici: "Es importante la unión de los movimientos porque la situación va a ser muy fea". El Salto. Disponible en: https://www.elsaltodiario.com/mecambio/encuentro-con-silvia-federici

Dietz, Mary. (2005). Las discusiones actuales de la teoría feminista. Debate Feminista, 32(16), 179-224. Disponible en: https://debatefeminista.cieg.unam.mx/index.php/category/vol-32/

Escobar, Arturo. (2003). «Mundos y conocimientos de otro modo». El programa de investigación de modernidad/colonialidad latinoamericano, Tabula Rasa. (1), 51-86.

Esquivel, Valeria. (2013). Los cuidados en los hogares y las comunidades. Documento Conceptual. Informe de Investigación. OXFAM International.

Federici, Silvia. (2013). Revolución en punto cero. Trabajo doméstico, reproducción y luchas feministas (Carlos Fernández Guervós y Paula Martín Ponz, Trads.). España: Traficantes de Sueños (Obra original publicada en 2012).

Flórez, Julia. (2010). Lecturas emergentes. Decolonialidad y subjetividad en las teorías de movimientos sociales. Bogotá: Pontificia Universidad Javeriana.

Fraser, Nancy. (2016). Las contradicciones del capital y los cuidados. New Left review (100), 111-132. Disponible en: http://www.rebelion.org/docs/223186.pdf

Fraser, Nancy; Arruzza, Cinzia y Bhattacharya, Tithi. (2019). Feminismo para el 99\%. Un Manifiesto. España: Herder Editorial. 
Gago, Veronica [En movimiento teve]. (20 de octubre de 2016). Paro nacional de mujeres [Archivo de video]. Disponible en: https://www.facebook.com/enmovimiento.teve/videos/788455791190896/?t=27

Gargallo, Francesca. (2019). Ideas y prácticas del entre-mujeres a principios del siglo XXI. Colombia: Ediciones Desde abajo.

Heller, Agnes. (1988). Los movimientos culturales como vehículo de cambio. Nueva Sociedad (96)39-49.

Herrero, Yeyo. (2013). Miradas ecofeministas para transitar a un mundo justo y sostenible. Revista de Economía Crítica (16), 278-307.

Lamus, Doris. (2010). De la subversión a la inclusión: Movimientos de mujeres de la segunda ola en Colombia, 1975-2005. Bogotá: Instituto de Antropología e Historia ICANH-.

Lamus, Doris. (2012). El color negro de la (sin) razón blanca: el lugar de las mujeres afrodescendientes en los procesos organizativos en Colombia. Universidad Autónoma de Bucaramanga. Bucaramanga, Colombia.

Lamus, Doris. (21 de junio de 2018). El siglo de las mujeres. La Silla Vacía. Disponible en: https://lasillavacia.com/silla-llena/red-de-las-mujeres/historia/el-siglo-de-las-mujeres-66709

Lamus, Doris. (Julio, 2020). “Genealogías de los feminismos. Escuela de Formación Mujer, Poder y Política, Módulo 2. Fundación Mujer y Futuro-HBS, Bucaramanga, Colombia.

Libre pensamiento. (2017). Feminismos (91) 1-92. CGT. Disponible en: http://cgt.org.es/secciones/social/libre-pensamiento-n\%C2\%BA-91-en-pdf-feminismos

Lospennato, Silvia [Resistiendo con aguante]. (junio 14 de 2018). sesión Cámara de Diputados - 13 de junio de 2018 -Ley de despenalizacion del aborto [Archivo de video]. Disponible en: https://www.youtube.com/watch?v=NcPG9jwxy7A

Meloni, Carolina. (2012). Las fronteras del feminismo. Teorías nómadas, mestizas y postmodernas. España: Editorial fundamentos.

Möller, Nicole. (8 de junio de 2018). “¿Que todo Chile se vuelva feminista!”. Marx21. Disponible en: https://marx21.net/2018/06/08/chile-feminista/

La Red 21. (28 de junio de 2009). Países con sufragio femenino (por fecha de aprobación). La Red 21. Disponible en: http://www.lr21.com.uy/mujeres/371172-paises-con-sufragiofemenino-por-fecha-de-aprobacion 
Piketty, Thomas. (2014). El capitalismo en el siglo XXI. Cambridge, Massachusetts: Harvard University Press.

Rodríguez, Corina. (2018). Los aportes de la economía feminista a la agenda feminista en América Latina a la agenda global. En: Aportes a la economía feminista desde Argentina. Análisis No. 27. Friedrich Ebert Stiftung; Argentina.

Rodríguez, Rosa (Ed.). (2015). Sin género de dudas. Logros y desafíos del feminismo hoy. Madrid: Biblioteca Nueva.

Segato, Rita [L]. (22 de noviembre de 2018). Rita Segato en Foro CLACSO 2018. [Archivo de video]. Disponible en https://www.youtube.com/watch?v=e_b7TC1Jbto\&fbclid=IwAR3XYtUSo6CvGB3IJe5 KvdR4px_yyfglUdzv1B6NX-1U0TUg3Iu_tFaxKWs

Suárez, Danila. (14 de diciembre de 2018). Ahora que sí nos leen: Filosofía y feminismo en América Latina. Economía Femini(s)ta $(E F)$. Disponible en: https://economiafeminita.com/ahora-que-si-nos-leen-filosofia-y-feminismo-en-americalatina/

Toca, María. (1 de septiembre de 2019). Entrevista a Rosa Cobo Bedia. La Pajarera magazine. Disponible en: http://www.lapajareramagazine.com/entrevista-a-rosa-cobobedia

BBC New. (13 de agosto de 2019). Violaciones en CDMX: los 2 casos de adolescentes supuestamente agredidas sexualmente por policías que indignan a la capital de México. $B B C$ New. Disponible en: https://www.bbc.com/mundo/noticias-america-latina49339317

Villegas, Paula. (10 de marzo de 2020) Las mujeres de México toman las calles para protestar contra la violencia. The New York Time. Disponible en: https://www.nytimes.com/es/2020/03/10/espanol/mexico-paro-mujeres-protestas.html 